\title{
Why Should Gastroenterologists Know About Fibromyalgia? Common Pathogenesis and Clinical Implications
}

\author{
Yong Sung Kim \\ Wonkwang Digestive Disease Research Institute, Wonkwang University School of Medicine, Iksan, Jeollabuk-do, Korea
}

Article: Validation of new symptom-based fibromyalgia criteria for irritable bowel syndrome co-morbidity studies Sperber AD, Akiva S, Leshno M, Halpern Z, Buskila D

(J Neurogastroenterol Motil 2011;17:67-72)

Patients with irritable bowel syndrome (IBS) frequently present non-gastrointestinal (GI) somatic disorders about twice as much as a control population. ${ }^{1}$ Fibromyalgia (FM), chronic fatigue syndrome, temporomandibular joint disorder and chronic pelvic pain are the best documented non-GI somatic comorbidities. ${ }^{1}$ The presence of comorbidities in patients with IBS can lead to decrease quality of life, increase health care seeking and increase medical cost. ${ }^{2}$

FM, the most frequently investigated comorbidity of IBS, is a soft tissue disorder characterized by diffuse musculoskeletal pain and specific tender points on examination. ${ }^{3}$ Its prevalence is estimated around $2 \%$ in the general population and up to $20 \%$ among rheumatology outpatients. ${ }^{1,4}$ However, the prevalence of FM in patients with IBS is approximately $32.5 \%$ (26\%-65\%) and the prevalence of IBS in patients with FM is approximately $48 \%(32 \%-77 \%){ }^{1-3}$

The high prevalence of non-GI comobidites in IBS suggests that there might be common pathophysiologic mechanisms in IBS and comorbidities such as abnormal pain sensitivity and descending pain inhibition, autonomic nervous system dysregulation, brain-gut axis dysfunction, immune dysfunction and abnormalities in the levels of serotonin or its receptor. ${ }^{1,2,5}$ In addition, psychosocial factors including psychological stress, emotional stress and emotional, physical or sexual abuse are trigger factors in both IBS and FM. ${ }^{5}$ However, these possible common underlying pathophysiologic mechanisms have not been proven yet. ${ }^{2}$

Although IBS and FM are widely overlapped, gastroenterologists usually do not pay attention to FM or other non-GI comorbidities when they diagnose or manage patients with IBS. And the researchers of IBS clinical trials also have not considered this problem when they set the study population or analyze the therapeutic effects of certain drugs. In contrast, physicians who deal with FM (eg, neurologist, primary care physician, pain specialist and rheumatologist etc) considered IBS and pain/cramp in abdomen as one of 10 most relevant to a diagnosis of FM. ${ }^{6}$ Especially, it is difficult to diagnose or assess FM in the GI clinic

Received: September 24, 2010 Revised: September 26, 2010 Accepted: September 28, 2010

(c) This is an Open Access article distributed under the terms of the Creative Commons Attribution Non-Commercial License (http://creativecommons. org/licenses/by-nc/3.0) which permits unrestricted non-commercial use, distribution, and reproduction in any medium, provided the original work is properly cited.

*Correspondence: Yong Sung Kim, MD, PhD

Wonkwang Digestive Disease Research Institute, Wonkwang University Hospital, 344-2 Shinyong-dong, Iksan, Jeollabuk-do 570-711, Korea

Tel: +82-63-859-2563, Fax: +82-63-855-2025, E-mail: wms89@hanmail.net

Financial support: This work was supported by Wonkwang University, 2010.

Conflicts of interest: None. 
because it requires a trained rheumatologist to perform physical examination according to American College of Rheumatology criteria.

In this issue of the Journal, Sperber et al demonstrated new symptom-based FM criteria that can be used in IBS patients without physical examination. This study should be welcomed because there are several points that gastroenterologists should consider about FM in diagnosis and management of IBS.

First, IBS patients with co-morbid FM might be a new separate IBS subgroup. Riedl et al $^{2}$ suggest that patients with IBS could be categorized according to existence of co-morbidity and hypothesized that psychological factors play a common etiological role in IBS and co-morbidities. Patients with a high degree of somatic co-morbidity also present higher levels of anxiety and depression, as well as a more pronounced decrease in quality of life and higher intensity of abdominal pain/discomfort. ${ }^{7}$ Because usual conventional management for IBS symptoms could not improve global symptoms in this patients group, it is necessary to identify the IBS patient with co-morbid FM and to establish a new treatment strategy with optimal consultation.

Second, FM may contribute the female predominance of IBS. Sperber et al recruited only female patients in this study and it was based on the fact that the IBS or FM is a predominantly female disorder. It was one of the major limitations of this study. Then, what about the sex difference according to co-morbid FM in patients with IBS? Akkuş et al ${ }^{8}$ performed a comparative study addressing the effects of female predominance of FM on the female-to-male ratios of IBS. There was a significant female predominance in patients with IBS + FM (F:M = 5:1), but the proportion of male is higher than that of female in patients with IBS only ( $\mathrm{F}: \mathrm{M}=2: 3)$. They postulated that coexisting FM increases the female proportion of IBS patients. Although it needs to be confirmed in a large study, we should keep FM in mind when we see female patients with IBS.

Third, co-morbid FM could be a confounding factor in clinical trials of therapeutic drug for IBS. Because common pathogenesis is present in IBS and FM, treatment for IBS could affect not only abdominal pain/discomfort but also symptoms of FM in IBS patient with co-morbid FM. Low serum level of serotonin and tryptophan, low cerebrospinal fluid level of serotonin metabolite and increased density of serotonin receptors on circulating platelets have been found in patients with FM. ${ }^{5,9}$ Therefore we can postulate that serotonergic drug used in treatment of IBS could have an effect on symptoms of FM, however many clinical trials of serotonergic drugs usually ignored this possibility.
Although studied with a small number, $5-\mathrm{HT}_{4}$ agonist, tegaserod, improved not only constipation predominant IBS status but also FM impact questionnaire score. ${ }^{9}$ However, the correlation between changes in constipation predominant IBS status and FM impact questionnaire score was relatively low and insignificant. The 5- $\mathrm{HT}_{3}$ antagonist, ondansetron and tropistron, also improved pain intensity in studies with patients with FM. ${ }^{10,11}$ Another interesting finding is the presence of small intestinal bacterial overgrowth (SIBO) in patient with FM. Pimentel et $\mathrm{al}^{12}$ reported that an abnormal breath test was higher in FM (100\%) than control $(20 \%)$ or even IBS (84\%). Furthermore, significantly greater hydrogen production was noted in FM than IBS and peak hydrogen level correlated with the degree of somatic pain. Although there have been no direct evidence that eradication of SIBO can improve symptoms of FM, in a study with IBS patients with co-morbid restless legs syndrome that frequently occurs in FM, restless legs syndrome was significantly improved after rifaximin and tegaserod therapy or rifaximin monotherapy. $^{13}$

Interestingly, despite of author's worry about the propensity for reporting pain in IBS patients, IBS patients exhibited relatively low sensitivity of new symptom-based criteria. This unexpected result might be related to low number of IBS group, therefore it needs to be investigated in a large number of IBS patients.

In summary, the very significant study by Sperber et al validated new symptom-based FM criteria in a population including IBS and FM patients for the first time. Although further validation studies with a large number of patients with IBS in different races and cultures are still needed, this study gave us a useful tool to approach FM more easily in the GI clinic. In the future, gastroenterologists could identify a new IBS subgroup, establish a new therapeutic strategy and modify the methodical design in clinical trials by using new symptom-based FM criteria.

\section{References}

1. Whitehead WE, Palsson O, Jones KR. Systematic review of the comorbidity of irritable bowel syndrome with other disorders: what are the causes and implications? Gastroenterology 2002;122:1140-1156.

2. Riedl A, Schmidtmann M, Stengel A, et al. Somatic comorbidities of irritable bowel syndrome: a systematic analysis. J Psychosom Res 2008;64:573-582.

3. Sperber AD, Dekel R. Irritable bowel syndrome and co-morbid gastrointestinal and extra-gastrointestinal functional syndromes. J Neurogastroenterol Motil 2010;16:113-119.

4. Goldenberg DL. Fibromyalgia and related syndromes. In: Klippel 
JH, Dieppe PA, eds. Rheumatology. Philadelphia: Mosby 1998: 1-12.

5. Bradley LA. Pathophysiology of fibromyalgia. Am J Med 2009; 122(12 suppl):S22-S30.

6. Silverman SL, Harnett J, Zlateva G, Mardekian J. Identifying fibromyalgia-associated symptoms and conditions from a clinical perspective: a step toward evaluating healthcare resource utilization in fibromyalgia. Pain Pract 2010;10:520-529.

7. Vandvik PO, Wilhelmsen I, Ihlebaek C, Farup PG. Comorbidity of irritable bowel syndrome in general practice: a striking feature with clinical implications. Aliment Pharmacol Ther 2004;20:1195-1203.

8. Akkuş S, Senol A, Ayvacioglu NB, Tunc E, Eren I, Isler M. Is female predominance in irritable bowel syndrome related to fibromyalgia? Rheumatol Int 2004;24:106-109.

9. Reitblat T, Zamir D, Polishchuck I, Novochatko G, Malnick S, Kalichman L. Patients treated by tegaserod for irritable bowel syn- drome with constipation showed significant improvement in fibromyalgia symptoms. A pilot study. Clin Rheumatol 2009;28:10791082 .

10. Hrycaj P, Stratz T, Mennet P, Müller W. Pathogenetic aspects of responsiveness to ondansetron (5-hydroxytryptamine type 3 receptor antagonist) in patients with primary fibromyalgia syndrome - a preliminary study. J Rheumatol 1996;23:1418-1423.

11. Späth M, Stratz T, Färber L, Haus U, Pongratz D. Treatment of fibromyalgia with tropisetron - dose and efficacy correlations. Scand J Rheumatol 2004;33(suppl 119):63-66.

12. Pimentel $\mathrm{M}$, Wallace $\mathrm{D}$, Hallegua $\mathrm{D}$, et al. A link between irritable bowel syndrome and fibromyalgia may be related to findings on lactulose breath testing. Ann Rheum Dis 2004;63:450-452.

13. Weinstock LB, Fern SE, Duntley SP. Restless legs syndrome in patients with irritable bowel syndrome: response to small intestinal bacterial overgrowth therapy. Dig Dis Sci 2008;53:1252-1256. 